\title{
Cholesterol biosynthesis inhibitor RO 48-8071 inhibits pancreatic ductal adenocarcinoma cell viability by deactivating the JNK and ERK/MAPK signaling pathway
}

\author{
ZHEN DING ${ }^{1,2 *}$, YANAN GU ${ }^{3 *}$, DAKE HUANG ${ }^{4 *}$, HONG ZHOU $^{3}$, TINGTING ZHU $^{3}$,

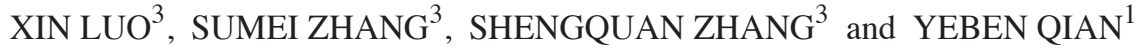 \\ ${ }^{1}$ Department of General Surgery, The First Affiliated Hospital of Anhui Medical University, \\ Hefei, Anhui 230022; ${ }^{2}$ Department of General Surgery, The Affiliated Chaohu Hospital of Anhui Medical University, \\ Chaohu, Anhui 238000; ${ }^{3}$ Department of Biochemistry and Molecular Biology; ${ }^{4}$ Comprehensive Laboratory, \\ School of Basic Medical Sciences, Anhui Medical University, Hefei, Anhui 230032, P.R. China
}

Received February 13, 2021; Accepted July 12, 2021

DOI: $10.3892 / \mathrm{mmr} .2021 .12468$

\begin{abstract}
The morbidity and mortality of pancreatic cancer have been continuously increasing, causing seven deaths per 100,000 individuals/year. At present, effective therapies are severely lacking, thus, highlighting the importance of developing novel therapeutic approaches. The present study aimed to investigate the inhibitory roles of the 2,3-oxidosqualene cyclase inhibitor, RO 48-8071 (RO), on pancreatic ductal adenocarcinoma. RO was used to treat the pancreatic cancer cell line (PANC-1) in vitro to examine the effects of $\mathrm{RO}$ on cell viability, as well as to determine its potential molecular mechanism. Moreover, experiments in a xenograft model of subcutaneous tumors generated by injecting PANC-1 cells hypodermically into nude mice were performed to observe the inhibition of RO on tumor growth. It was found that RO inhibited PANC-1 cell viability when treatment was given for 24, 48 and $72 \mathrm{~h}$. The in vivo study demonstrated that RO markedly inhibited subcutaneous tumor growth in nude mice. Further studies revealed that $\mathrm{RO}$ could induce cell cycle arrest in the $\mathrm{G}_{1}$ phase by regulating p27, cyclin B1 and cyclin E expression to inhibit PANC-1 cell viability. Moreover, RO inactivated
\end{abstract}

Correspondence to: Professor Shengquan Zhang, Department of Biochemistry and Molecular Biology, School of Basic Medical Sciences, Anhui Medical University, 81 Meishan Road, Hefei, Anhui 230032, P.R. China

E-mail: zhangshengquan@ahmu.edu.cn

Dr Yeben Qian, Department of General Surgery, The First Affiliated Hospital of Anhui Medical University, 218 Jixi Road, Hefei, Anhui 230022, P.R. China

E-mail: qianyeben@hotmail.com

*Contributed equally

Key words: pancreatic ductal adenocarcinoma, proliferation, viability, RO 48-8071 the JNK and ERK MAPK signaling pathway by decreasing the phosphorylation levels of JNK and ERK. Collectively, the present study demonstrated that RO served anti-pancreatic cancer roles in vitro and in vivo, which may provide new ideas and facilitate the development of novel treatment options for pancreatic cancer.

\section{Introduction}

Pancreatic cancer, of which the most common histological subtype is pancreatic ductal adenocarcinoma (PDAC), has worldwide incidence and mortality rates of eight cases per 100,000 person/year and seven deaths per 100,000 person/year, respectively (1). By 2017, pancreatic cancer had become the third-leading cause of cancer-associated mortality in the United States. The incidence and mortality of pancreatic cancer are continuing to increase, and it will be the second largest cause of cancer-related mortality worldwide by 2030, based on predicted data (2). Pancreatic cancer is an aggressive and fatal disease, and most patients with pancreatic cancer succumb to the disease within 5 years (3). The 5-year overall survival rate is $8 \%$, but in patients with advanced disease, this decreases to $3 \%$ (4). The causes of the poor prognosis of patients with pancreatic cancer include a lack of early symptoms, no clinically validated screening methods in the early stage and early invasion of nearby vessels, amongst other factors $(3,5)$. For pancreatic cancer, the only current treatment option is surgery. However, only $10-15 \%$ of newly diagnosed patients qualify for surgery, as pancreatic cancer is often in the late stage when diagnosed $(3,5,6)$.

Currently, therapies for pancreatic cancer are seriously lacking. Combination chemotherapies, which have been previously used for the treatment of patients with pancreatic cancer, such as Folfirinox and gemcitabine/nab-paclitaxel, have only extended the patients' survival by several months and cause evident toxic side effects $(7,8)$. In addition, immunotherapy has not been shown to be effective against PDAC. It has also been revealed that whole-cell therapeutic vaccines show no effect in patients with advanced-stage pancreatic cancer $(9,10)$. 
Therefore, more effective and less toxic drugs are urgently required for patients with pancreatic cancer.

An increasing number of studies have revealed that the activation of the mevalonate pathway or cholesterol intake via low density lipoprotein receptor (LDLR) is associated with the development of pancreatic cancer (10-12). High expression levels of 3-hydroxy-3-methylglutaryl-coenzyme A (HMG-CoA) reductase and LDLR were observed in a PDAC mouse model (10). These findings suggested that cholesterol level could influence the development of PDAC. Cholesterol is a basic and important functional and structural element of cell membranes, and it served a key role in cell differentiation, proliferation and apoptosis $(2,6,13)$. In the process of synthesizing cholesterol from cytoplasmic acetyl-CoA, HMG-CoA reductase catalyzes the conversion of HMG-CoA to mevalonate, and this is the rate-limiting step of the pathway (14). Moreover, inhibitors of HMG-CoA reductase can inhibit the proliferation of some cancer cells, such as breast cancer cells and ovarian cancer cells, by blocking cholesterol biosynthesis (15-17).

The conversion of 2, 3-monoepoxysqualene to lanosterol, which is catalyzed by a key enzyme 2, 3-oxidosqualene cyclase (OSC), is a critical step downstream of HMG-CoA reductase in the process of cholesterol biosynthesis (14). OSC has become a feasible new target for inhibiting cholesterol biosynthesis (18). RO 48-8071 (RO) is an inhibitor of OSC (19). It has been reported that RO has an inhibitory effect on the proliferation of prostate and breast cancer cells $(20,21)$. Inhibitors of cholesterol synthesis can regulate apoptosis, angiogenesis and metastasis via the ERK and JNK signaling pathway in breast and pancreatic cancer $(22,23)$. The present study conducted a set of experiments to investigate the effects of $\mathrm{RO}$ on pancreatic cancer and to determine the possible molecular mechanisms.

\section{Materials and methods}

Reagents. RO was purchased from MedChemExpress (cat. no. HY-18630A) and dissolved in DMSO with a storage concentration of $10 \mathrm{mM}$. DMEM was purchased from Gibco (Thermo Fisher Scientific, Inc.), while FBS was obtained from Clark Bioscience. Specific antibodies against $\beta$-actin, p27, phosphorylated (p)ERK1/2, ERK1/2, pJNK, JNK, cyclin B1 and cyclin E were purchased from Santa Cruz Biotechnology, Inc. A specific antibody for Ki67 immunohistochemical staining was obtained from OriGene Technologies, Inc. HRP-conjugated secondary antibodies were obtained from MilliporeSigma, and used for western blotting. HRP-conjugated secondary antibodies and a DAB HRP Color Development kit for immunohistochemistry were purchased from OriGene Technologies, Inc.

Cell culture. The human pancreatic tumor cell line PANC-1 was purchased from the American Type Culture Collection. Cells were maintained routinely in complete DMEM (DMEM with $10 \%$ FBS) with $1 \%$ penicillin-streptomycin in a $5 \% \mathrm{CO}_{2}$ humidified atmosphere at $37^{\circ} \mathrm{C}$.

Animals. A total of 12 male BALB/c nude mice aged 4 weeks (weight, 16.86 $\pm 9.3 \mathrm{~g}$ ) for the in vivo study were provided by
Beijing Vital River Laboratory Animal Technology Co., Ltd., and reared according to the Animal Research Committee's guidelines of Anhui Medical University (Hefei, China). The mice were raised in the specific-pathogen-free laboratory animal room under humane conditions at $22 \pm 2^{\circ} \mathrm{C}$ with $55 \pm 5 \%$ humidity under a 12-h light/dark cycle with food and water provided ad libitum. The animal protocols were approved by the Committee for Ethics of Animal Experimentation of Anhui Medical University (approval no. 20150136).

Cell viability assay. An MTS assay (Promega Corporation) was performed to assess cell viability. Following trypsinization, $\sim 2,000$ PANC-1 cells in the logarithmic growth phase were prepared as a unicellular suspension to seed in a 96-well cell culture plate with $100 \mu \mathrm{l}$ volume in triplicate. Cells were treated with $\mathrm{RO}$ at different concentrations $(1,3,10,30$ and $100 \mu \mathrm{M})$ for $72 \mathrm{~h}$ when they reached $60-70 \%$ confluence. Then, the MTS reagent was added to the 96 -well cell culture plate $(20 \mu 1$ per well), which was followed by incubation at $37^{\circ} \mathrm{C}$ for $2 \mathrm{~h}$. The absorbance value (A value) at the wavelength of $490 \mathrm{~nm}$ was measured on a universal microplate reader (ELx800; BioTek Instruments, Inc.), with six samples measured in each group. Using this method, an appropriate concentration of RO for the treatment of PANC-1 cells was determined and used in the subsequent experiments. $\mathrm{RO}$ at the selected concentration was used to treat PANC-1 cells as aforementioned for 0, 24, 48 and $72 \mathrm{~h}$ to detect the time-dependent effects of RO on PANC-1 cell viability.

Cell cycle analysis via flow cytometry (FCM). PANC-1 cells at a density of $1 \times 10^{5}$ cells per well were inoculated into 6-well cell culture plates and cultured in a $5 \% \mathrm{CO}_{2}$ humidified atmosphere at $37^{\circ} \mathrm{C}$. After treatment with RO (24, 48 and $72 \mathrm{~h}$ ) when cells reached 60-70\% confluence, a Cell Cycle Analysis kit (Beyotime Institute of Biotechnology) was used to detect the cell cycle, according to the manufacturer's instructions. Briefly, the cells were collected and counted after trypsin digestion. Then, cells were fixed in cold $70 \%$ ethanol for $30 \mathrm{~min}$ at $4^{\circ} \mathrm{C}$ and stained with PI in the dark for 20 min after washing twice with cold PBS. The cell cycle was then assessed via FCM (BD FACSVerse ${ }^{\mathrm{TM}}$; BD Biosciences). Data analysis was performed using FlowJo software (FlowJo version 10.5.4; FlowJo LLC).

Western blotting. PANC-1 cells were treated with RO for 24, 48 and $72 \mathrm{~h}$, and were harvested in RIPA lysis buffer $(25 \mathrm{mM}$ Hepes; $1.5 \%$ Triton X-100; $1 \%$ sodium deoxycholate; $0.1 \%$ SDS; $0.5 \mathrm{M} \mathrm{NaCl} ; 5 \mathrm{mM}$ EDTA; $50 \mathrm{mM} \mathrm{NaF} ; 0.1 \mathrm{mM}$ sodium vanadate; $1 \mathrm{mM}$ phenylmethylsulphonyl fluoride; $0.1 \mathrm{~g} / 1$ leupeptin; $\mathrm{pH}$ 7.8) on ice. Cell lysates were centrifuged at $14,000 \mathrm{x} \mathrm{g}$ for $30 \mathrm{~min}$ at $4^{\circ} \mathrm{C}$ to obtain the total soluble protein by collecting the supernatant. The concentration of the extracted proteins from each group was determined using a BCA assay (Beyotime Institute of Biotechnology). Then, $30 \mu \mathrm{g} /$ lane of total protein from each treatment group, which had been boiled together with SDS-PAGE loading buffer, was loaded onto $12 \%$ SDS-PAGE gels and subsequently transferred onto PVDF membranes after electrophoresis separation. The blots were run on separate gels simultaneously by loading the same concentrations of the proteins derived from the same sample 
into each gel. After non-specific blocking in 5\% skimmed milk for $2 \mathrm{~h}$ at room temperature, the PVDF membranes were washed with PBS containing 0.1\% Tween-20 three times and incubated at $4{ }^{\circ} \mathrm{C}$ overnight with specific primary antibodies against $\beta$-actin (cat. no. sc-47778; 1:1,000), cyclin B1 (cat. no. sc-245; 1:500), cyclin E (cat. no. sc-377100; 1:500), p27 (cat. no. sc-56338; 1:500), pERK (cat. no. sc-81492; 1:500), pJNK (cat. no. sc-6254; 1:500), ERK1/2 (cat. no. sc-514302; $1: 1,000)$ and JNK (cat. no. sc-7345; 1:1,000). Subsequently, the membranes were reacted with the corresponding HRP-conjugated secondary antibody (goat anti-mouse IgG; cat. no. AP130P; 1:10,000) diluted in TBS solution for $2 \mathrm{~h}$ at room temperature. After another washing step to remove unbound secondary antibodies, the specific bands were developed using ECL reagents (SuperSignal ${ }^{\mathrm{TM}}$ West Femto kit; Pierce; Thermo Fisher Scientific, Inc.), and images were captured using a ChemiScope (Clinx Science Instruments Co., Ltd.). The signals of the specific bands were identified and detected with the Quantity One analysis software (version 4.6.6; Bio-Rad Laboratories, Inc.). Target bands for cyclin B1, cyclin E and p27 were normalized to the reference gene $\beta$-actin, and specific pERK and pJNK bands were normalized to total ERK1/2 or JNK, respectively.

In vivo experiment. For the in vivo tumorigenic study, 12 male $\mathrm{BALB} / \mathrm{c}$ nude mice were inoculated subcutaneously in the right hips with $1 \times 10^{6}$ PANC-1 cells suspended in PBS. When the tumors reached $0.4-0.5 \mathrm{~cm}$, the mice were injected with $50 \mu \mathrm{l} \mathrm{RO}$ at a concentration of $5 \mathrm{mg} / \mathrm{kg} /$ day or DMSO at a relevant concentration ( $n=6$ for each group) via the vena cava caudalis once a day for the first 5 days, then once every other day for six more injections (20). The tumor volumes were measured every 3 days at day $0,3,6,9,12,15,18$ and 21 of RO injection. The tumor size was calculated as $a b^{2} / 2$, where $a$ was the longer diameter and $b$ was the smaller diameter of the two dimensions. The maximum tumor size obtained in the present study was $\sim 250 \mathrm{~mm}^{3}$. At day 24 after RO injection, the mice were anesthetized with an intraperitoneal injection of pentobarbital $(50 \mathrm{mg} / \mathrm{kg})$ and euthanized via cervical dislocation. Surgery for subcutaneous tumor dissection was performed to obtain the formed xenograft tumors.

$H \& E$ and tissue immunohistochemical staining. The excised xenograft tumors were immediately fixed in $10 \%$ formalin for $24 \mathrm{~h}$ at room temperature, followed by dehydration in a gradient series of ethanol and paraffin embedding. Formalin-fixed and paraffin-embedded tissues were sliced (4- $\mu \mathrm{m}$ thick) and conventionally stained with hematoxylin for $5 \mathrm{~min}$ at room temperature and eosin for $10 \mathrm{sec}$ at room temperature. The H\&E-stained sections were visualized using a light microscope (magnification, $\mathrm{x} 400$ ) after sealing the slides with neutral balsam.

For immunohistochemical staining, the sections $(4-\mu \mathrm{m}$ thick) obtained from the formalin-fixed and paraffin-embedded tissues were blocked with 3\% BSA (Beyotime Institute of Biotechnology) diluted in $0.2 \%$ Triton X-100/PBS at room temperature for $30 \mathrm{~min}$ and stained with a Ki67 specific antibody (cat. no. ZA-0502; OriGene Technologies, Inc.) overnight at $4^{\circ} \mathrm{C}$, followed by incubation with HRP-conjugated secondary antibody (cat. no. PV-6001) for $2 \mathrm{~h}$ at room temperature and development with a DAB Horseradish Peroxidase Color Development kit (cat. no. ZLI-9018; OriGene Technologies, Inc.). The images of the stained sections were captured using a light microscope (magnification, x400; Olympus Corporation).

Statistical analysis. All data were analyzed using SPSS version 15.0 software (SPSS, Inc.). All data are presented as the mean \pm SEM ( $n=3$ for the in vitro assays and $n=6$ mice/group for the in vivo assays). The significant differences between groups were determined using one-way ANOVA followed by a Tukey's post hoc test. $\mathrm{P}<0.05$ was considered to indicate a statistically significant difference.

\section{Results}

RO inhibits the viability of PANC-1 cells. PANC-1 cells in the 96-well culture plate were treated with $\mathrm{RO}$ at a variety of concentrations $(1,3,10,30$ and $100 \mu \mathrm{M})$ for $72 \mathrm{~h}$ to determine cell viability using a colorimetric MTS assay. It was found that RO inhibited PANC-1 cell viability in a dose-dependent manner (Fig. 1A). RO showed a nearly $40 \%(\mathrm{P}<0.05)$ inhibitory ratio at $10 \mu \mathrm{M}$, and an $80 \%$ inhibitory ratio $(\mathrm{P}<0.05)$ at 30 and $100 \mu \mathrm{M}$ after treatment for $72 \mathrm{~h}$. However, at 1 and $3 \mu \mathrm{M}$ concentration, RO did not have an effect on the viability of PANC-1 cells.

It was demonstrated that the effects of RO on PANC-1 cell viability were in a time-dependent manner. The growth inhibition percentages were $4 \%(\mathrm{P}<0.05), 17 \%(\mathrm{P}<0.05)$ and $42 \%(\mathrm{P}<0.05)$ when PANC-1 cells were treated with $10 \mu \mathrm{M}$ RO for 24, 48 and 72 h, respectively (Fig. 1B). In accordance with these results from the MTS assay, RO at a concentration of $10 \mu \mathrm{M}$ was selected to treat PANC-1 cells in the subsequent experiments.

$R O$ inhibits the $G_{1}$-S-phase transition of PANC-1 cells. The influence of RO on the cell cycle phase distribution of PANC-1 cells was determined via FCM after treatment with $10 \mu \mathrm{M}$ RO for different durations. It was identified that RO treatment for $72 \mathrm{~h}$ increased $\mathrm{G}_{1}$ phase arrest from $51.57 \%$ ( $0 \mathrm{~h}$ control treatment group) to $88.76 \%$, and reduced the $\mathrm{S}$ and $\mathrm{G}_{2}$ phase percentages from 27.48 and $20.6 \%$ to 7.66 and $3.81 \%$, respectively (Fig. 2).

$R O$ regulates the expression levels of cell cycle-related genes and the phosphorylation levels of ERK and JNK in PANC-1 cells. In order to understand how RO could cause cell cycle arrest at the $G_{1}$ phase, the expression of cell cycle-related proteins, cyclin B1, cyclin E and p27 were measured in RO-treated cells. The results suggested that RO treatment decreased the expression levels of cyclin B1 and cyclin E (Fig. 3). However, RO increased the expression levels of p27.

The MAPK signaling pathway serves an important role in controlling cellular biological behavior, such as cell proliferation, apoptosis and migratory potential (24). Thus, the phosphorylation levels of ERK and JNK were determined to observe whether RO exerts its roles via the ERK and JNK MAPK signaling pathway. It was found that RO reduced the expression levels of pERK $1 / 2$ and pJNK in the PANC- 1 cells, which also had a constant level of ERK1/2 and JNK expression (Fig. 4). 

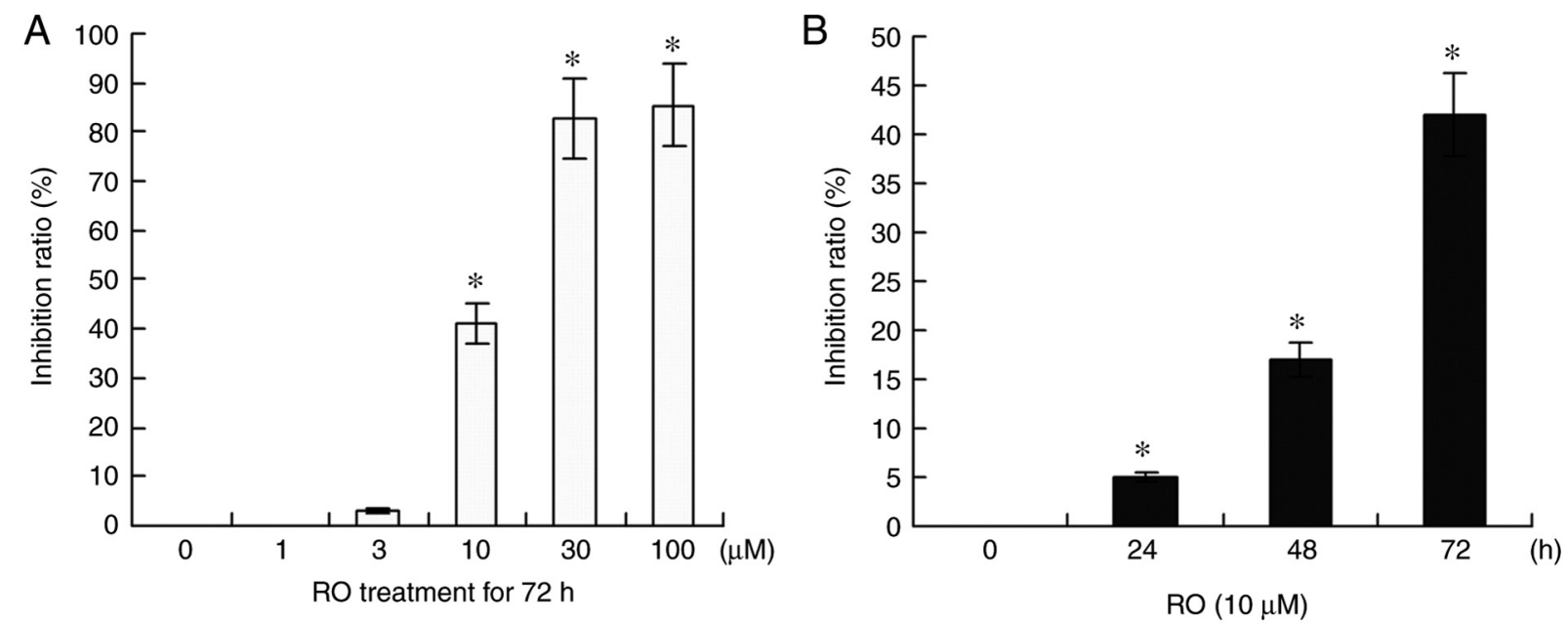

Figure 1. RO inhibits the viability of PANC-1 cells. Cells were treated with RO at (A) different doses and for (B) different durations. The inhibition ratio of each group was detected using an MTS assay. ${ }^{~} \mathrm{P}<0.05$ vs. control group. RO, RO 48-8071.

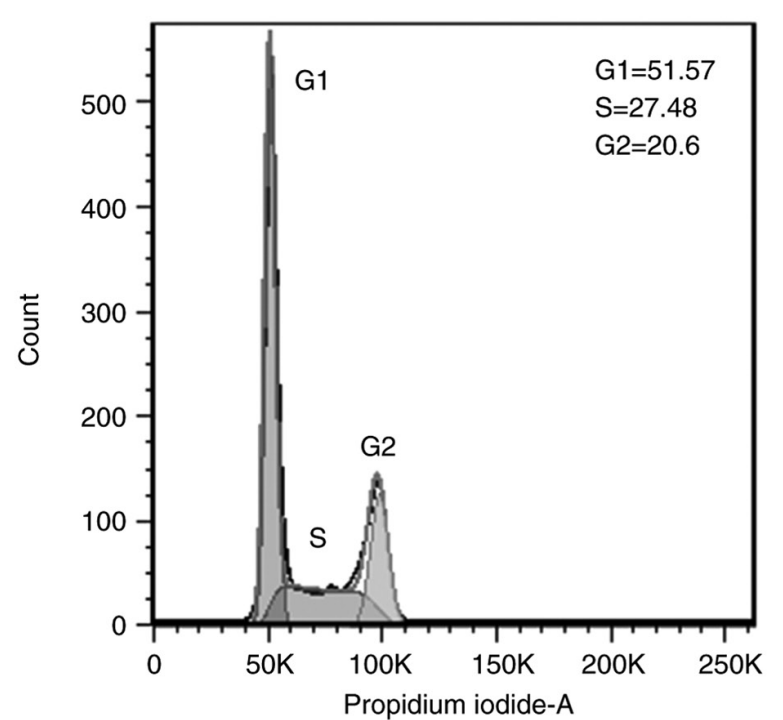

$\mathrm{Oh}$

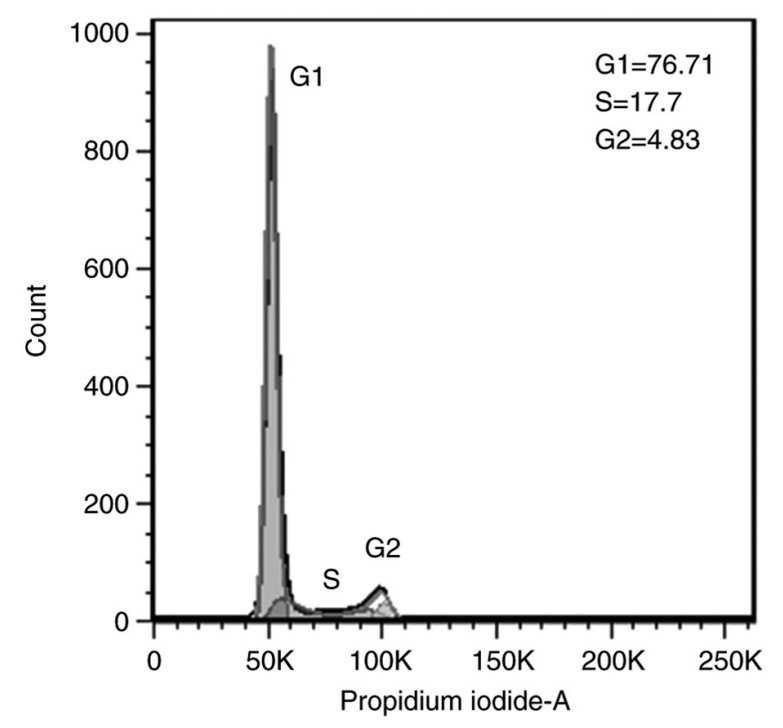

$48 \mathrm{~h}$

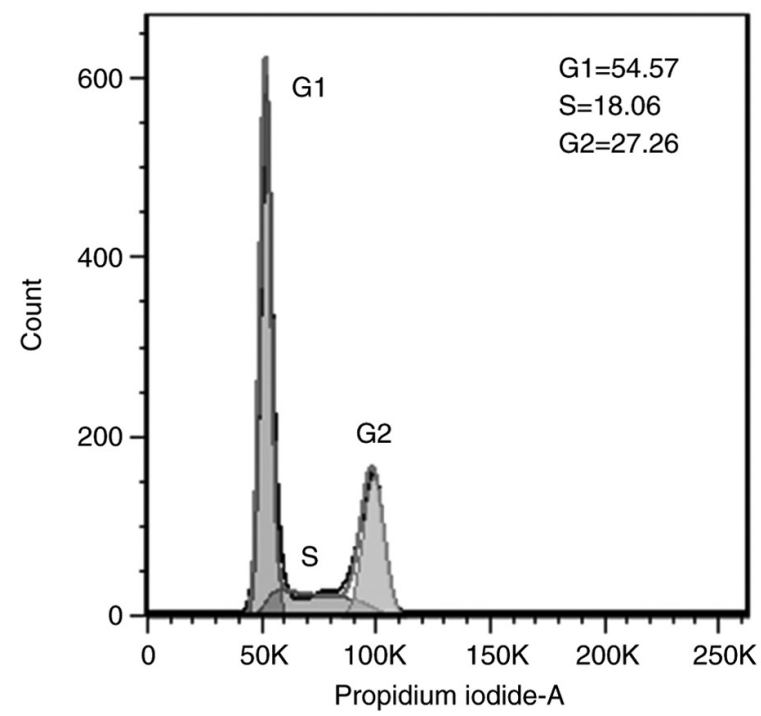

$24 \mathrm{~h}$

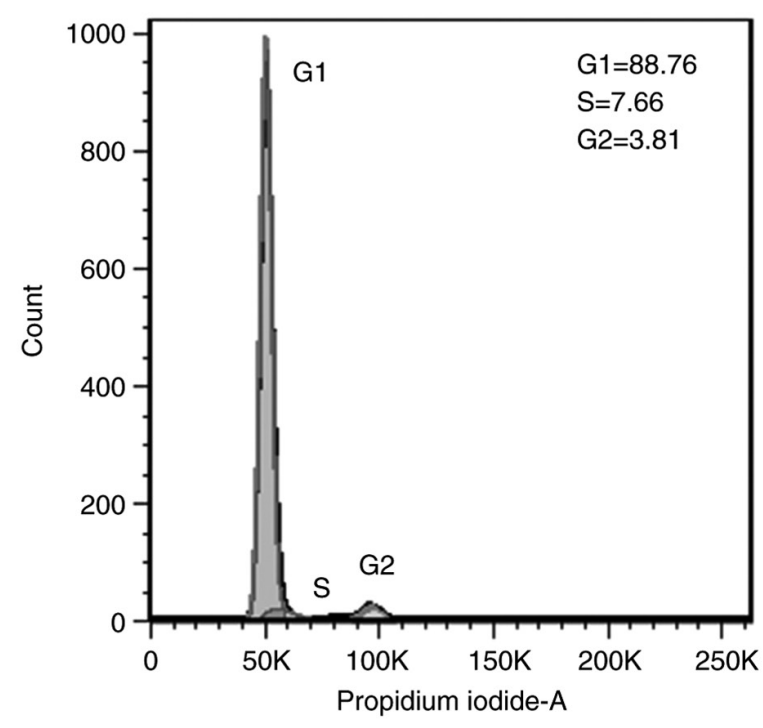

$\mathrm{RO}(10 \mu \mathrm{M})$

Figure 2. RO inhibits the $\mathrm{G}_{1}-\mathrm{S}$-phase transition of PANC-1 cells. Cells were treated with RO $(10 \mu \mathrm{M})$ for 24,48 and $72 \mathrm{~h}$. Cell cycle analysis was performed using flow cytometry. The results represent one of three independent experiments. RO, RO 48-8071. 


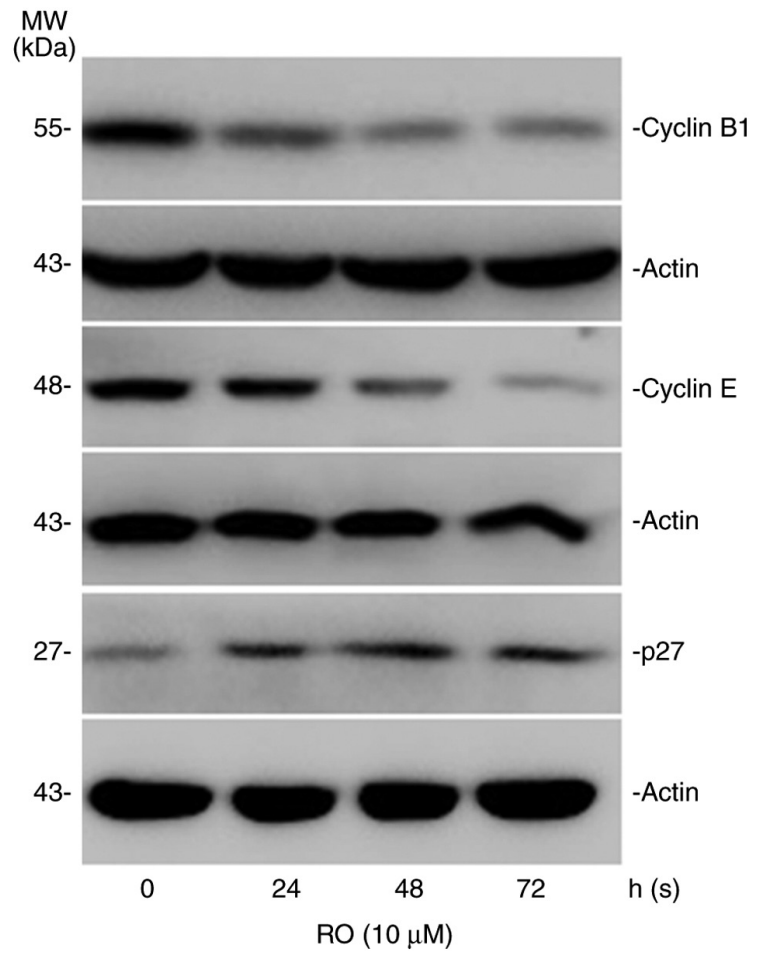

Figure 3. RO regulates the expression levels of cell cycle-related proteins. PANC-1 cells were cultured and treated with RO $(10 \mu \mathrm{M})$ for 24,48 and $72 \mathrm{~h}$. The cells were harvested to detect the expression levels of cell cycle-related proteins via western blotting. RO, RO 48-8071.

RO inhibits the oncogenicity of PANC-1 cells in vivo. In order to further confirm the inhibitory effects of RO on pancreatic cancer in vivo, a xenograft model of subcutaneous tumor growth was established in nude mice. The experimental mice were sacrificed by cervical dislocation after the xenografts were treated with RO via intravenous injection for 3 weeks. The tumor volumes were measured every 3 days at day $0,3,6$, $9,12,15,18,21$ and 24 of RO injection, and the tumor volumes shown as the ordinate are the average tumor volume of each mouse. As presented in Fig. 5A-D, the weights and volume of tumors formed by RO-treated PANC-1 cells were significantly lower and smaller compared with those of the control tumors. Moreover, Ki67-specific staining of the tumor tissue sections from the xenograft animal model revealed that RO treatment markedly inhibited cell proliferation in vivo, which was demonstrated by a weaker staining of Ki67 when compared with the control group (Fig. 6).

\section{Discussion}

In total, 85,100 individuals died from pancreatic cancer in 2016, which has become the third-leading cause of cancer-associated mortality worldwide, with a 5-year overall survival rate of $8 \%$ (4), mainly due to a lack of efficacious methods to treat or control pancreatic cancer. The reasons for the poor prognosis of pancreatic cancer include uncontrolled and excessive proliferation, and early invasion $(3,5)$. The main strategy of tumor treatment is to effectively suppress the malignant behaviors of tumor cells.

High levels of HMG-CoA reductase and LDLR have been observed in a PDAC mouse model, and that LDLR-mediated

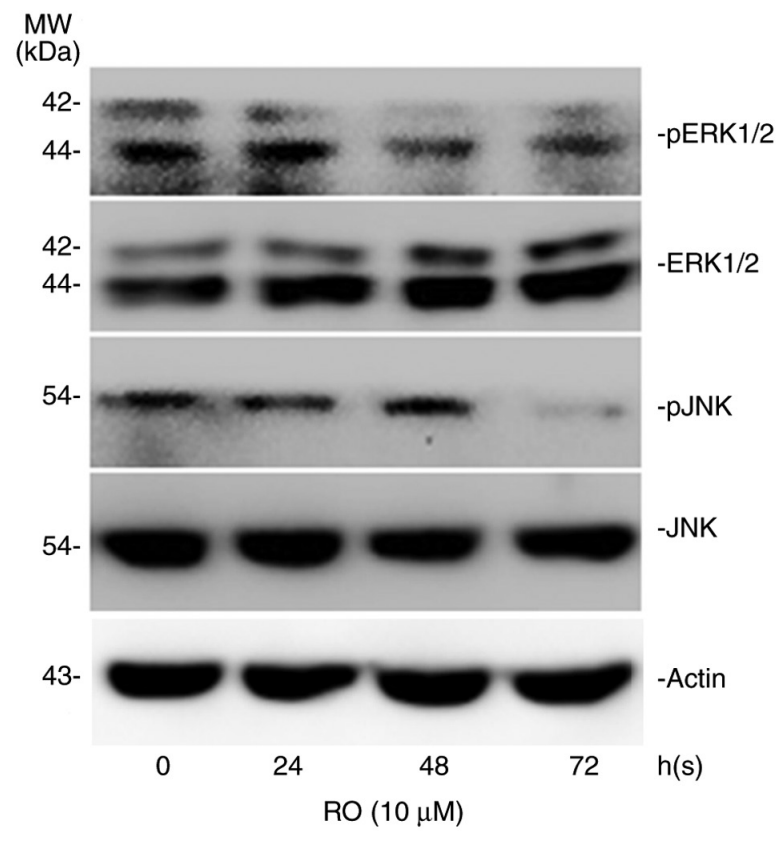

Figure 4. RO regulates the phosphorylation of ERK and JNK.PANC-1 cells were cultured and treated with RO $(10 \mu \mathrm{M})$ for 24,48 and $72 \mathrm{~h}$. The cells were harvested to detect the phosphorylation levels of ERK and JNK, which were normalized to total ERK and JNK. RO, RO 48-8071; p, phosphorylated.

activation of the mevalonate pathway or cholesterol intake is associated with the development of pancreatic cancer (10-12), which suggests that cholesterol levels may influence the development of PDAC. Cholesterol is important in the regulation of cellular processes, such as cell proliferation, differentiation and apoptosis $(2,6,13)$. In the process of synthesizing cholesterol from cytoplasmic acetyl-CoA, HMG-CoA reductase catalyzes the conversion of HMG-CoA to mevalonate, which is the rate-limiting step of the pathway (14). Inhibitors of HMG-CoA reductase can inhibit the proliferation of some cancer cells by blocking cholesterol biosynthesis (15-17). It has been shown that statins, which are inhibitors of HMG-CoA reductase, can also be used to treat cancer (25). However, statins cause a shortage of numerous downstream intermediate products, including farnesyl pyrophosphate and geranylgeranyl diphosphate, which are involved in the roles of isoprenoids, and membrane structure and function, and thus produces considerable side effects (26). The conversion of 2,3-monoepoxysqualene to lanosterol by OSC is a key step that is downstream of $\mathrm{HMG}-\mathrm{CoA}$ reductase in the process of cholesterol biosynthesis. Previously, RO, an inhibitor of OSC, has become a possible novel target to inhibit cholesterol biosynthesis $(17,18)$. Furthermore, RO may cause fewer side effects than statins, as it primarily only decreases the level of cholesterol, without decreasing the levels of other intermediate products of the mevalonate pathway (21).

It has been reported that RO 48-8071 has an inhibitory effect on the proliferation of prostate and breast cancer cells $(20,21,27)$ and leukemia cells $(28)$. The present study also demonstrated its inhibitory roles on pancreatic cancer development and provided insights into the potential molecular mechanisms of RO. Moreover, the current study found notable inhibitory effects of RO on pancreatic cancer cells (PANC-1) in vitro. It was also identified that RO markedly inhibited cell 
A
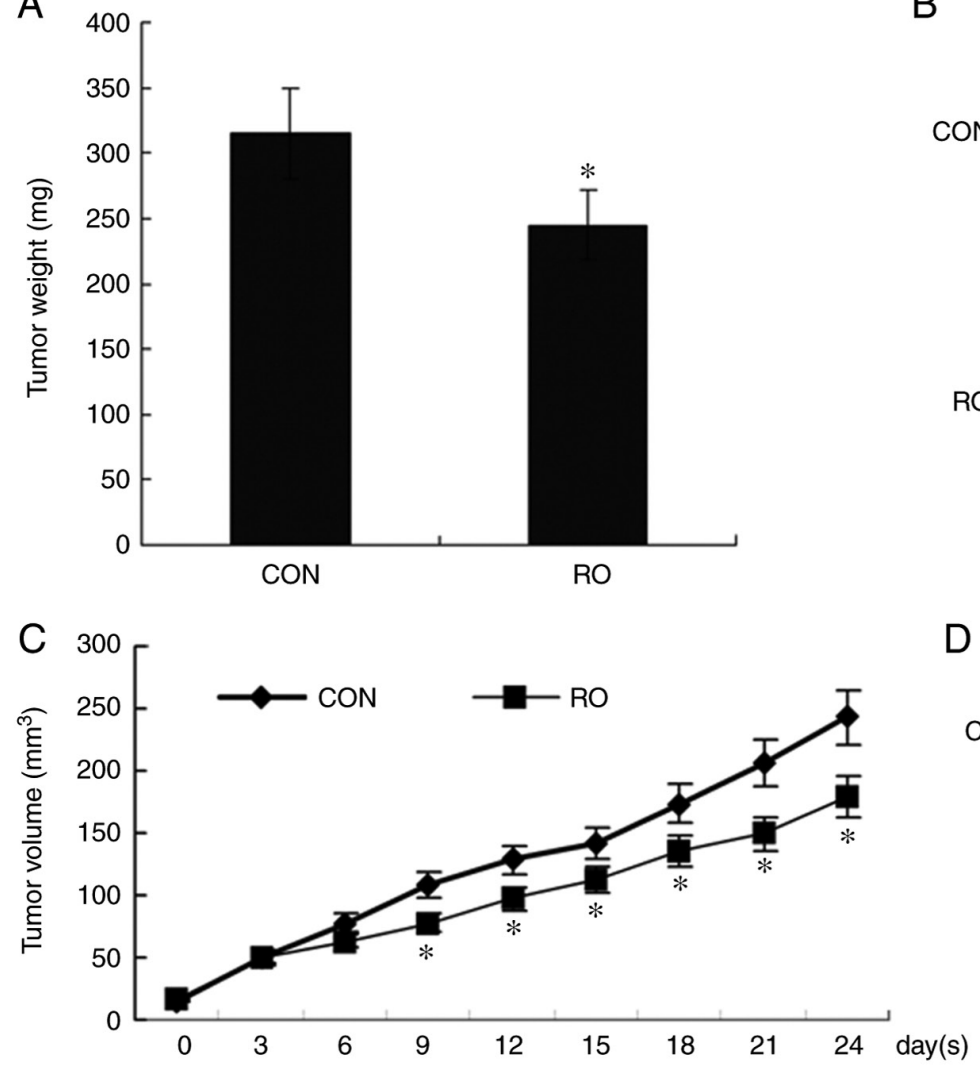

B

CON

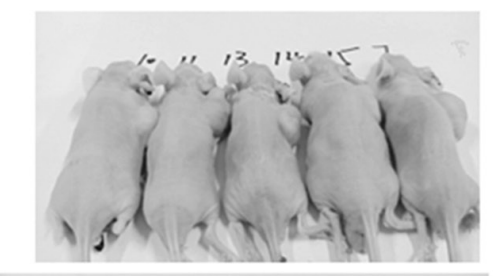

RO
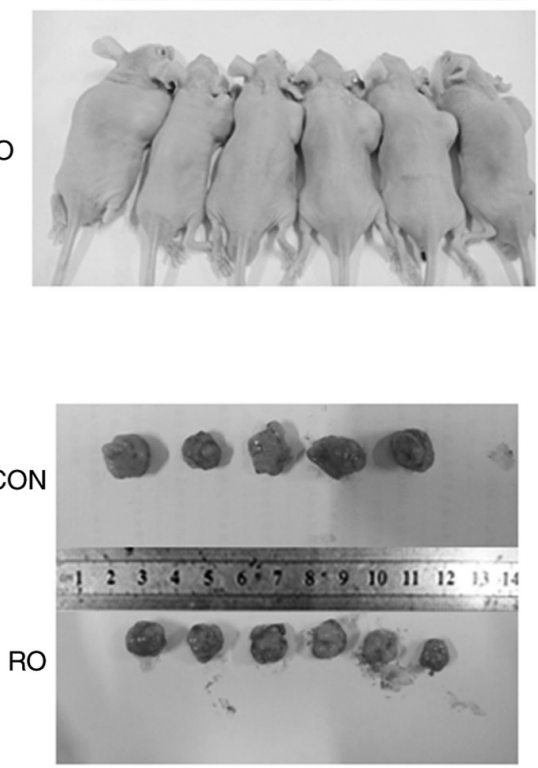

Figure 5. RO inhibits tumor growth in nude mice. An in vivo experiment was performed to examine the inhibitory effect of RO on tumor growth. Male $\mathrm{BALB} / \mathrm{c}$ nude mice were inoculated subcutaneously in the right flanks with PANC-1 cells to form xenograft tumors, followed by intravenous injection with RO or DMSO (as CON). The tumor volumes were measured every 3 days at days $0,3,6,9,12,15,18,21$ and 24 . (A) Tumors weights of the two groups. (B) Representative images of tumor-bearing mice. (C) Tumor volume analysis. The tumor volumes shown as the ordinate are the average tumor volume of each mouse. (D) Resected xenograft tumors from the two groups. "P<0.05 vs. CON group. CON, control; RO, RO 48-8071.

proliferation in vivo, as shown by a lower Ki67 expression in xenograft tissues.

Cyclin B and cyclin E, which are commonly known factors involved in $\mathrm{G}_{1} / \mathrm{S}$ cell cycle-regulation, interact with CDKs to stimulate the transition from the $G_{1}$ phase to the $S$ phase, while p27 acts as a CDK inhibitor to inhibit the activities of cyclin/CDK complexes (29). The overexpression of cyclin B and cyclin E appear to be correlated with high tumor grade in breast cancer (30). By detecting the cell cycle and cell viability influenced by RO, the present study found that RO caused $\mathrm{G}_{1}$ phase arrest and inhibited cell viability by regulating the expression levels of the cell cycle-related proteins p27, cyclin B1 and cyclin E. This was consistent with previous findings concerning the action of RO on other cancer cells $(20,21)$. It is commonly known that cholesterol is a component of the cell structure. A noteworthy hypothesis is that RO causes a shortage of cholesterol, which consequently causes the inhibition of cell viability (31). The present results revealed that RO mediated the suppression of $G_{1} / S-C D K$ activity and the activation of the CDK inhibitor $\mathrm{p} 27$, which caused replicative arrest, and this could be one of the mechanisms of action of the antitumor roles of RO.

The MAPK signaling pathways are widely known to be involved in multiple cell behaviors and are associated with tumor development. ERK is an important mitogen-activated proliferation factor that can promote cell survival, whereas the JNK/MAPK pathway serves key roles in apoptotic processes (32). The ERK

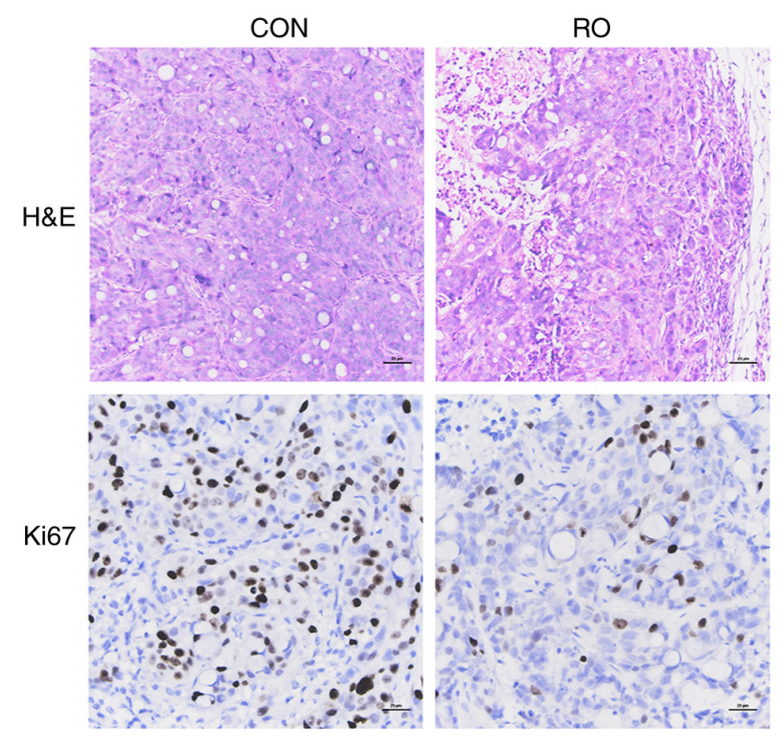

Figure 6. RO inhibits the expression level of the proliferation marker protein Ki67 in excised xenograft tumors. The tissue sections were conventionally stained with H\&E or stained using a specific Ki67 antibody. The upper panel shows representative images of H\&E staining and the bottom panel shows representative images of Ki67 immunohistochemical staining. Scale bar, $25 \mu \mathrm{m}$. CON, control; RO, RO 48-8071

and JNK/MAPK signaling pathways are often overactivated in most types of cancer cells and serve a key role in controlling cell 
proliferation, apoptosis and motility $(33,34)$. Cholesterol depletion on the membrane surface can inhibit PI3K/Akt and ERK pathways in several types of tumors (35). RO 48-8071 has been reported to inhibit ERK phosphorylation in both HCT116 and HPAF-II cells (23). The present mechanistic studies revealed that RO reduced the expression level of pERK1/2 and $\mathrm{pJNK}$, which suggested that RO inactivated the ERK and JNK signaling pathway in PANC-1 cells.

In conclusion, the present results demonstrated that the anti-pancreatic cancer effects of RO occurred via the regulation of the cell cycle to inhibit the viability of pancreatic cancer cells. RO may exert these roles by regulating the expression levels of cell cycle-related proteins, concomitantly with the inhibition of the ERK and JNK pathway. A preliminary conclusion could be drawn from the data obtained in the present study that RO could be used as an antitumor reagent to limit the development of pancreatic cancer. Due to its low side effects, RO may facilitate the treatment of pancreatic cancer. The effects of RO on cell viability and the cell cycle identified in the present study could be due to either a RO-induced decreased in cholesterol level or mechanisms that are independent of the cholesterol levels. Moreover, RO could be used to exert a direct effect on tumor cells. The major limitation of the present study is that only one cell line was used. Further in-depth studies using multiple pancreatic cancer cell lines are necessary to clarify and provide details on the specific underlying mechanisms.

\section{Acknowledgements}

Not applicable.

\section{Funding}

The present study was supported by grants from the General Program of National Natural Science Foundation of China (grant no. 81271748) and the University Science Research Project of Anhui Province (grant nos. KJ2020A0143 and KJ2017A195).

\section{Availability of data and materials}

The datasets used and/or analyzed during the current study are available from the corresponding author on reasonable request.

\section{Authors' contributions}

ShZ and YQ designed the study and revised the manuscript. $\mathrm{ZD}$ and YG performed the majority of the experiments. $\mathrm{DH}$ performed the histological examination and interpreted the data. YG and SuZ analyzed and interpreted the data, and wrote and revised the manuscript. HZ, TZ and XL analyzed and interpreted the data. ShZ and YQ confirm the authenticity of all the raw data. All authors have read and approved the final manuscript.

\section{Ethics approval and consent to participate}

The animals involved in the present study were reared according to the Animal Research Committee's guidelines of Anhui Medical University (Hefei, China). The animal protocols were approved by the Committee for Ethics of Animal Experimentation of Anhui Medical University (approval no. 20150136).

\section{Patient consent for publication}

Not applicable.

\section{Competing interests}

The authors declare that they have no competing interests.

\section{References}

1. Xiao AY, Tan MLY, Wu LM, Asrani VM, Windsor JA, Yadav D and Petrov MS: Global incidence and mortality of pancreatic diseases: A systematic review, meta-analysis, and meta-regression of population-based cohort studies. Lancet Gastroenterol Hepatol 1: 45-55, 2016.

2. Rahib L, Smith BD, Aizenberg R, Rosenzweig AB, Fleshman JM and Matrisian LM: Projecting cancer incidence and deaths to 2030: The unexpected burden of thyroid, liver, and pancreas cancers in the United States. Cancer Res 74: 2913-2921, 2014

3. Siegel RL, Miller KD and Jemal A: Cancer Statistics, 2017. CA Cancer J Clin 67: 7-30, 2017.

4. American Cancer Society: Cancer Facts \& Figures 2017. American Cancer Society, 2017.

5. Makohon-Moore A and Iacobuzio-Donahue CA: Pancreatic cancer biology and genetics from an evolutionary perspective. Nat Rev Cancer 16: 553-565, 2016.

6. Kleeff J, Korc M, Apte M, La Vecchia C, Johnson CD, Biankin AV, Neale RE, Tempero M, Tuveson DA, Hruban RH and Neoptolemos JP: Pancreatic cancer. Nat Rev Dis Primers 2: $16022,2016$.

7. Von Hoff DD, Ervin T, Arena FP, Chiorean EG, Infante J, Moore M, Seay T, Tjulandin SA, Ma WW, Saleh MN, et al: Increased survival in pancreatic cancer with nab-paclitaxel plus gemcitabine. N Engl J Med 369: 1691-1703, 2013.

8. Vaccaro V, Sperduti I and Milella M: FOLFIRINOX versus gemcitabine for metastatic pancreatic cancer. N Engl J Med 365: 768-769, 2011.

9. Morrison AH, Byrne KT and Vonderheide RH: Immunotherapy and prevention of pancreatic cancer. Trends Cancer 4: 418-428, 2018.

10. Guillaumond F, Bidaut G, Ouaissi M, Servais S, Gouirand V, Olivares O, Lac S, Borge L, Roques J, Gayet O, et al: Cholesterol uptake disruption, in association with chemotherapy, is a promising combined metabolic therapy for pancreatic adenocarcinoma. Proc Natl Acad Sci 112: 2473-2478, 2015.

11. Chen H, Qin S, Wang M, Zhang T and Zhang S: Association between cholesterol intake and pancreatic cancer risk: Evidence from a meta-analysis. Sci Rep 5: 8243, 2015.

12. Sumi S, Beauchamp RD, Townsend CM Jr, Uchida T, Murakami M, Rajaraman S, Ishizuka J and Thompson JC: Inhibition of pancreatic adenocarcinoma cell growth by lovastatin. Gastroenterology 103: 982-989, 1992.

13. Neoptolemos JP, Palmer DH, Ghaneh P, Psarelli EE, Valle JW, Halloran CM, Faluyi O, O'Reilly DA, Cunningham D, Wadsley $\mathrm{J}$, et al: European study group for pancreatic cancer. Comparison of adjuvant gemcitabine and capecitabine with gemcitabine monotherapy in patients with resected pancreatic cancer (ESPAC-4): A multicentre, open-label, randomised, phase 3 trial. Lancet 389: 1011-1024, 2017.

14. IkonenE:Cellularcholesterol trafficking and compartmentalization. Nat Rev Mol Cell Biol 9: 125-138, 2008.

15. Cuccioloni M, Bonfili L, Mozzicafreddo M, Cecarini V, Scuri S, Cocchioni M, Nabissi M, Santoni G, Eleuteri AM and Angeletti M: Mangiferin blocks proliferation and induces apoptosis of breast cancer cells via suppression of the mevalonate pathway and by proteasome inhibition. Food Funct 7: 4299-4309, 2016.

16. Yeganeh B, Wiechec E, Ande SR, Sharma P, Moghadam AR, Post M, Freed DH, Hashemi M, Shojaei S, Zeki AA and Ghavami S: Targeting the mevalonate cascade as a new therapeutic approach in heart disease, cancer and pulmonary disease. Pharmacol Ther 143: 87-110, 2014. 
17. Kato S, Liberona MF, Cerda-Infante J, Sánchez M, Henríquez J, Bizama C, Bravo ML, Gonzalez P, Gejman R, Brañes J, et al: Simvastatin interferes with cancer 'stem-cell' plasticity reducing metastasis in ovarian cancer. Endocr Relat Cancer 25: 821-836, 2018.

18. Charlton-Menys V and Durrington PN: Squalene synthase inhibitors: Clinical pharmacology and cholesterol-lowering potential. Drugs 67: 11-16, 2007.

19. Morand OH, Aebi JD, Dehmlow H, Ji YH, Gains N, Lengsfeld H and Himber J: RO 48-8.071, a new 2,3-oxidosqualene: Lanosterol cyclase inhibitor lowering plasma cholesterol in hamsters, squirrel monkeys, and minipigs: Comparison to simvastatin. J Lipid Res 38: 373-390, 1997.

20. Liang Y, Mafuvadze B, Aebi JD and Hyder SM: Cholesterol biosynthesis inhibitor RO 48-8071 suppresses growth of hormone-dependent and castration-resistant prostate cancer cells. Onco Targets Ther 9: 3223-3232, 2016.

21. Liang Y, Besch-Williford C, Aebi JD, Mafuvadze B, Cook MT, Zou X and Hyder SM: Cholesterol biosynthesis inhibitors as potent novel anti-cancer agents suppression of hormone-dependent breast cancer by the oxidosqualene cyclase inhibitor RO 48-8071. Breast Cancer Res Treat 146: 51-62, 2014

22. Gopalan A, Yu W, Sanders BG and Kline K: Simvastatin inhibition of mevalonate pathway induces apoptosis in human breast cancer cells via activation of JNK/CHOP/DR5 signaling pathway. Cancer Lett 329: 9-16, 2013

23. Maione F, Oliaro-Bosso S, Meda C, Di Nicolantonio F, Bussolino F, Balliano G, Viola F and Giraudo E: The cholesterol biosynthesis enzyme oxidosqualene cyclase is a new target to impair tumour angiogenesis and metastasis dissemination. Sci Rep 5: 9054, 2015.

24. Sun Y, Liu WZ, Liu T, Feng X, Yang N and Zhou HF: Signaling pathway of MAPK/ERK in cell proliferation, differentiation, migration, senescence and apoptosis. J Recept Signal Transduct Res 35: 600-604, 2015.

25. Garcia-Ruiz C, Morales A and Fernandez-Checa JC: Statins and proteinprenylation in cancer cell biology and therapy. Anticancer Agents Med Chem 12: 303-315, 2012
26. McTaggart SJ: Isoprenylated proteins. Cell Mol Life Sci 63: 255-267, 2006

27. Grinter SZ, Liang Y, Huang SY, Hyder SM and Zou X: An inverse docking approach for identifying new potential anti-cancer targets. J Mol Graph Model 29: 795-799, 2011.

28. Vilimanovich U, Bosnjak M, Bogdanovic A, Markovic I, Isakovic A, Kravic-Stevovic T, Mircic A, Trajkovic V and Bumbasirevic V: Statin-mediated inhibition of cholesterol synthesis induces cytoprotective autophagy in human leukemic cells. Eur J Pharmacol 765: 415-428, 2015.

29. Kaldis $\mathrm{P}$ and Aleem E: Cell cycle sibling rivalry: Cdc2 vs. Cdk2. Cell Cycle 4: 1491-1494, 2005.

30. Fan L, Cao X, Yan H, Wang Q, Tian X, Zhang L, He X, Borjihan G and Morigen: The synthetic antihyperlipidemic drug potassium piperate selectively kills breast cancer cells through inhibiting G1-S-phase transition and inducing apoptosis. Oncotarget 8: 47250-47268, 2017.

31. Sun Y, Sukumaran P, Varma A, Derry S, Sahmoun AE and Singh BB: Cholesterol-induced activation of TRPM7 regulates cell proliferation, migration, and viability of human prostate cells. Biochim Biophys Acta 1843: 1839-1850, 2014.

32. Johnson GL and Lapadat R: Mitogen-activated protein kinase pathways mediated by ERK, JNK, and p38 protein kinases. Science 298: 1911-1912, 2002.

33. Shen X, Cui X, Cui H, Jin Y, Jin W and Sun H: Geraniol and lupeol inhibit growth and promote apoptosis in human hepatocarcinoma cells through the MAPK signaling pathway. J Cell Biochem 120: 5033-5041, 2019.

34. Liu X, Yang Q, Yan J, Zhang $X$ and Zheng M: LncRNA MNX1-AS1 promotes the progression of cervical cancer through activating MAPK pathway. J Cell Biochem 120: 4268-4277, 2019.

35. Fang Z, Tang Y, Fang J, Zhou Z, Xing Z, Guo Z, Guo X, Wang W, Jiao W, Xu Z and Liu Z: Simvastatin inhibits renal cancer cell growth and metastasis via AKT/mTOR, ERK and JAK2/STAT3 pathway. PLoS One 8: e62823, 2013.

This work is licensed under a Creative Commons Attribution-NonCommercial-NoDerivatives 4.0 International (CC BY-NC-ND 4.0) License. 